\title{
A Low-Order Partial Integrated Guidance and Control Scheme for Diving Hypersonic Vehicles to Impact Ground Maneuver Target
}

\author{
Tong An $\mathbb{D D}^{1}{ }^{1}$ JianHua Wang $\left(\mathbb{D},{ }^{2}\right.$ YuLong Pan $\mathbb{D}^{1},{ }^{1}$ and HaiShan Chen ${ }^{1}{ }^{1}$ \\ ${ }^{1}$ People's Liberation Army Air Force Early Warning Academy, Wuhan, Hubei, China \\ ${ }^{2}$ Department of Aerospace Science and Technology, Space Engineering University, Beijing, China
}

Correspondence should be addressed to JianHua Wang; wjh_nudt_2013@163.com

Received 2 July 2021; Accepted 26 August 2021; Published 8 September 2021

Academic Editor: Jie Chen

Copyright $@ 2021$ Tong An et al. This is an open access article distributed under the Creative Commons Attribution License, which permits unrestricted use, distribution, and reproduction in any medium, provided the original work is properly cited.

In this article, a low-order partial integrated guidance and control (PIGC) design method is proposed for diving hypersonic vehicles to impact ground maneuver target. A three-channel analytical model of body rates is deduced based on acceleration components of the hypersonic vehicle. By combining the analytical model of body rates and relative dynamic model between the hypersonic vehicle and target, three-channel commands of body rates are directly generated based on the extended state observer (ESO) technique, sliding mode control approach, and dynamic surface control theory in the guidance subsystem. In the attitude control subsystem, a sliding mode controller is designed to track the commands of body rates and generate commands of control surface fin deflections. By making full use of acceleration information of the hypersonic vehicle measured by the mounted accelerometer, the proposed PIGC design method provides a novel solution to compensate the unknown acceleration of the ground maneuver target. Besides, the order of design model is also reduced, and the design process is simplified. The effectiveness and robustness of the PIGC design method are verified and discussed by $6 \mathrm{DOF}$ simulation studies.

\section{Introduction}

The studies on the hypersonic vehicle are increasing due to its unique advantages, such as global rapid attack and ultrahigh-speed transportation. Therefore, it has rapidly become a focused research topic in aerospace domain [1]. The dive flight of hypersonic vehicles exhibits characteristics of fast time-varying, strong coupling, and nonlinearity. The coupling between the guidance loop and control loop is strengthened, which proposes higher requirements for the guidance and control (G\&C) system design.

Compared with the traditional separated design approach of the G\&C system, the integrated guidance and control (IGC) method can fully consider the coupling between centroid motion and rotational motion of the hypersonic vehicle. Besides, the IGC method can ensure the overall stability and improve the accuracy of design model as well as the overall performance of the G\&C system [2-4]. Based on the published literatures, the IGC design methods can be divided into three types:
(1) Single-channel/plane IGC scheme: the three-dimensional (3D) motion of the air vehicle is decomposed into different channels or planes, and the coupling between each channel/plane is regarded as a small amount, which is generally neglected. Hence, the design of the 3D IGC system is reduced to a single-channel or single-plane low-order IGC design process [5-8].

(2) Full-state IGC scheme: a full-state high-order IGC design model is established, which takes rotational motion model of the air vehicle and relative motion model between the air vehicle and target into account. The order of the design model is usually $8-10$, and the design model is generally transformed into a strict feedback form. Then, the backstepping control, dynamic surface control, or other control methods are used to solve the high-order IGC system. Hence, the IGC system design is transformed into a high-order nonlinear control problem [4, 9-14]. 
(3) PIGC scheme: the PIGC scheme is executed in the guidance and control loops. The two-loop controller structure is similar to the full-state single-loop controller structure under some conditions. The PIGC method takes body rates instead of acceleration components of the air vehicle as the virtual inputs. The control loop is designed to track the commands of body rates. The order of design model and the number of design parameters can be reduced by using this method [15-17].

The target impacted by air vehicles is often maneuverable, and its acceleration is difficult to acquire directly, which increases the complexity of the G\&C system design. In recent years, most studies on the design of the G\&C system for air vehicles to impact maneuver target are based on the full-state coupled high-order IGC design model, which contains angles of line-of-sight (LOS), rotational Euler angles of the air vehicle, three-channel body rates, and other motion state variables. The IGC system design is accomplished through the backstepping control method or dynamic surface control method, and the uncertainties containing acceleration components of the target are estimated through some techniques, such as ESO [18-24], sliding mode observer $[25,26]$, and adaptive law [27]. The effectiveness of this kind of design method has been verified by nonlinear simulation results in the published literatures. However, there are still several disadvantages in the above studies. Firstly, the single-channel/plane IGC system design does not fully consider the coupling between each channel/ plane. Secondly, the full-state coupled high-order 3D IGC systems are still based on the traditional separated design method of the G\&C system to some extent. The angle of attack, sideslip angle, and bank angle are still the bridges connecting the guidance loop and control loop. The complexity of the high-order design model brings difficulty to the IGC system design. Thirdly, there are still limited studies on the G\&C system design for hypersonic vehicles to impact maneuver target.

Taking the above problems into account, a low-order PIGC design method for diving hypersonic vehicles to impact ground maneuver target is proposed in this article. By deducing a three-channel analytical model of body rates based on acceleration components of the hypersonic vehicle, the integration mechanism of rotational motion model of the hypersonic vehicle and relative motion model between the hypersonic vehicle and target is exploited. By combining the analytical model of body rates, relative motion model between the hypersonic vehicle and target, and rotational dynamic model of the hypersonic vehicle, a low-order PIGC scheme is presented, and the design process can be distilled to two steps:

(1) Three-channel commands of body rates are generated in the guidance loop based on ESO, sliding mode control approach, and dynamic surface control theory.

(2) Commands of control surface fin deflections are generated in the control loop based on sliding mode control approach.
This novel low-order PIGC scheme can offer an innovative method to compensate the unknown acceleration information of the maneuver target based on the measured acceleration of the hypersonic vehicle and simplify the design process of the G\&C system. The content of this article is arranged as follows. Section 2 proposes the 6DOF motion model of the hypersonic vehicle and relative motion model between the hypersonic vehicle and target in dive phase. Section 3.1 deduces the three-channel analytical model of body rates based on acceleration components of the hypersonic vehicle. Section 3.2 presents the design process of the guidance subsystem. Section 3.3 provides the design process of the attitude control subsystem. Section 4 conducts simulation experiments to verify the effectiveness and robustness of the proposed PIGC design method using the generic hypersonic vehicle (GHV) model. Section 5 summarizes the conclusion of this article.

\section{Motion Models}

The hypersonic vehicle in dive phase has the characteristics of high speed and short flight distance. Hence, the following reasonable assumptions are proposed to simplify the study process: (1) the Earth's rotation is neglected, and the Earth is considered as a flat ground; (2) the mass of the hypersonic vehicle is uniformly distributed, and the influence of body deformation is neglected; (3) the impacts of errors caused by the unmodeled parts of design model are ignored; and (4) the hypersonic vehicle is in unpowered flight during the dive phase regardless of thrust. The denoted motion models in this section consist of centroid and rotational dynamic and kinematical equations of the hypersonic vehicle as well as relative motion equations between the hypersonic vehicle and target. The motion models are given by variables in the ground inertial coordinate system, the body coordinate system, the ballistic coordinate system, and the LOS coordinate system, which are defined in Yan [3].

2.1. Centroid Equations. The centroid dynamic equations of the hypersonic vehicle in the ballistic coordinate system are denoted as follows [3]:

$$
\left\{\begin{array}{l}
\dot{v}=-g \sin \theta-\frac{D}{m}, \\
\dot{\theta}=\frac{1}{m v}\left(-m g \cos \theta+L \cos \gamma_{V}-N \sin \gamma_{V}\right), \\
\dot{\sigma}=\frac{1}{m v \cos \theta}\left(-L \sin \gamma_{V}-N \cos \gamma_{V}\right),
\end{array}\right.
$$

where $v$ is the velocity magnitude of the hypersonic vehicle, $g$ is the gravitational acceleration, $\theta$ is the flight path angle, $\sigma$ is the heading angle, $\gamma_{V}$ is the bank angle, and $m$ is the mass of the hypersonic vehicle. $D, L$, and $N$ are the aerodynamic drag, lift, and side force, respectively, which are calculated by 


$$
\begin{aligned}
D & =q S C_{D}, \\
L & =q S C_{L}, \\
N & =q S C_{N},
\end{aligned}
$$

where $q=0.5 \rho v^{2}$ is the dynamic pressure, $\rho$ is the density of atmosphere, $C_{L}$ is the lift coefficient, $C_{D}$ is the drag coefficient, $C_{N}$ is the side force coefficient, and $S$ is the aerodynamic reference area of the hypersonic vehicle.

The centroid kinematical equations of the hypersonic vehicle in the ground inertial coordinate system are given by

$$
\left\{\begin{array}{l}
\dot{x}=v \cos \theta \cos \sigma, \\
\dot{y}=v \sin \theta, \\
\dot{z}=-v \cos \theta \sin \sigma,
\end{array}\right.
$$

where $x, y$, and $z$ are the components of position vector of the hypersonic vehicle in the ground inertial coordinate system.

2.2. Rotational Equations. The rotational dynamic equations of the hypersonic vehicle in the body coordinate system are denoted as

$$
\left\{\begin{array}{l}
\dot{\omega}_{x}=J_{x}^{-1} M_{x}+J_{x}^{-1}\left(J_{y}-J_{z}\right) \omega_{z} \omega_{y} \\
\dot{\omega}_{y}=J_{y}^{-1} M_{y}+J_{y}^{-1}\left(J_{z}-J_{x}\right) \omega_{x} \omega_{z}, \\
\dot{\omega}_{z}=J_{z}^{-1} M_{z}+J_{z}^{-1}\left(J_{x}-J_{y}\right) \omega_{y} \omega_{x}
\end{array}\right.
$$

where $\omega_{x}$ is the roll rate, $\omega_{y}$ is the yaw rate, and $\omega_{z}$ is the pitch rate. $J_{x}, J_{y}$, and $J_{z}$ are the roll, yaw, and pitch moments of inertia of the hypersonic vehicle, respectively. $M_{x}$ is the roll moment, $M_{y}$ is the yaw moment, and $M_{z}$ is the pitch moment, which are calculated by

$$
\begin{aligned}
& M_{x}=q S l_{x} m_{x}, \\
& M_{y}=q S l_{y} m_{y}, \\
& M_{z}=q S l_{z} m_{z},
\end{aligned}
$$

where $l_{x}, l_{y}$, and $l_{z}$ are the aerodynamic reference lengths with respect to the roll, yaw, and pitch channels, respectively. $m_{x}$ is the roll aerodynamic moment coefficient, $m_{y}$ is the yaw aerodynamic moment coefficient, and $m_{z}$ is the pitch aerodynamic moment coefficient.

The rotational kinematical equations of the hypersonic vehicle in the body coordinate system are provided by

$$
\left\{\begin{array}{l}
\dot{\varphi}=\omega_{y} \sin \gamma+\omega_{z} \cos \gamma, \\
\dot{\psi}=\left(\omega_{y} \cos \gamma-\omega_{z} \sin \gamma\right) \sec \varphi, \\
\dot{\gamma}=\omega_{x}-\left(\omega_{y} \cos \gamma-\omega_{z} \sin \gamma\right) \tan \varphi,
\end{array}\right.
$$

where $\varphi$ is the pitch angle, $\psi$ is the yaw angle, and $\gamma$ is the roll angle.

2.3. Relative Motion Equations. Figure 1 illustrates the schematic diagram of relative motion between the hypersonic vehicle and ground maneuver target. $O_{B}$ and $T$ represent the centroids of the hypersonic vehicle and target, respectively. $\mathrm{O}-X Y Z$ is the ground inertial coordinate

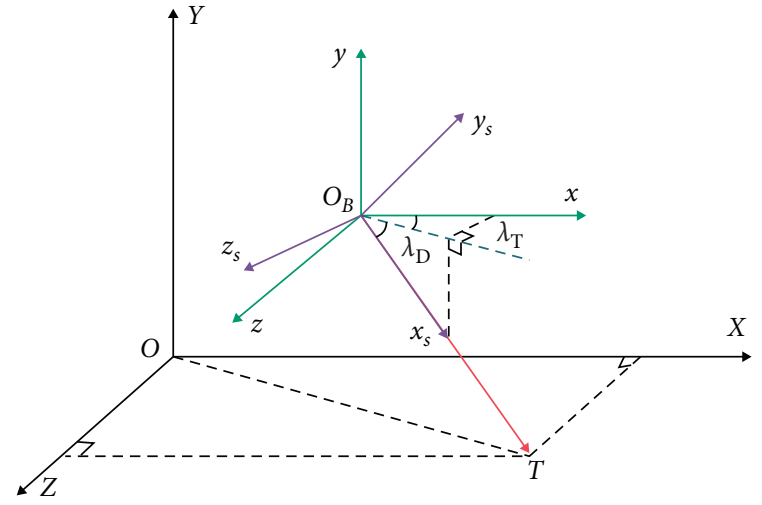

Figure 1: Schematic diagram of relative motion.

system. $O_{B}-x_{s} y_{s} z_{s}$ is the LOS coordinate system. The relative motion equations between the hypersonic vehicle and target are denoted as [3]

$$
\left\{\begin{array}{l}
\ddot{r}=r \dot{\lambda}_{D}^{2}+r \dot{\lambda}_{T}^{2} \cos ^{2} \lambda_{D}+a_{x s}^{T}-a_{x s}^{V}, \\
\ddot{\lambda}_{D}=\frac{\left(-2 \dot{\lambda}_{D}-r \dot{\lambda}_{T}^{2} \cos \lambda_{D} \sin \lambda_{D}\right)}{r}+\frac{\left(a_{y s}^{T}-a_{y s}^{V}\right)}{r}, \\
\ddot{\lambda}_{T}=\frac{\left(2 r \dot{\lambda}_{D} \dot{\lambda}_{T} \sin \lambda_{D}-2 \dot{r} \dot{\lambda}_{T} \cos \lambda_{D}\right)}{\left(r \cos \lambda_{D}\right)}-\frac{\left(a_{z s}^{T}-a_{z s}^{V}\right)}{\left(r \cos \lambda_{D}\right)},
\end{array}\right.
$$

where $r$ is the relative distance between the hypersonic vehicle and target, $\lambda_{D}$ is the elevation angle of LOS, $\lambda_{T}$ is the azimuth angle of LOS, $a_{x s}^{V}, a_{y s}^{V}$, and $a_{z s}^{V}$ are the acceleration components of the hypersonic vehicle in the LOS coordinate system, and $a_{x s}^{T}, a_{y s}^{T}$, and $a_{z s}^{T}$ are the acceleration components of the target in the LOS coordinate system.

\section{Partial Integrated Guidance and Control System Design}

In this section, the design scheme of a novel PIGC system is presented, which contains a guidance loop and a control loop. Firstly, a three-channel analytical model of body rates is deduced. Then, three-channel commands of body rates are generated in the guidance loop based on ESO, sliding mode control approach, and dynamic surface control theory. Finally, a sliding mode attitude controller is designed to track the commands of body rates, and commands of control surface fin deflections are directly obtained in the control loop.

3.1. Three-Channel Analytical Model of Body Rates. According to equation (3), the velocity vectors of the hypersonic vehicle denoted in the ground inertial coordinate system and the body coordinate system, respectively, hold the following relation:

$$
\left[\begin{array}{c}
v \cos \theta \cos \sigma \\
v \sin \theta \\
-v \cos \theta \sin \sigma
\end{array}\right]=\mathbf{T}_{\mathrm{GB}}\left[\begin{array}{c}
u \\
v \\
w
\end{array}\right],
$$


where $\left[\begin{array}{lll}u & v & w\end{array}\right]^{T}$ is the velocity vector of the hypersonic vehicle in the body coordinate system and $\mathrm{T}_{\mathrm{GB}}$ is the transition matrix from the body coordinate system to the ground inertial coordinate system. The derivatives of both sides of equation (8) can be written as

$$
\frac{d}{\mathrm{~d} t}\left(\left[\begin{array}{c}
v \cos \theta \cos \sigma \\
v \sin \theta \\
-v \cos \theta \sin \sigma
\end{array}\right]\right)=\frac{d}{\mathrm{~d} t}\left(\mathbf{T}_{\mathrm{GB}}\right)\left[\begin{array}{l}
u \\
v \\
w
\end{array}\right]+\mathbf{T}_{\mathrm{GB}} \frac{d}{\mathrm{~d} t}\left(\left[\begin{array}{c}
u \\
v \\
w
\end{array}\right]\right) .
$$

The left side of equation (9) holds the following relation:

$$
\frac{d}{\mathrm{~d} t}\left(\left[\begin{array}{c}
v \cos \theta \cos \sigma \\
v \sin \theta \\
-v \cos \theta \sin \sigma
\end{array}\right]\right)=\mathbf{T}_{\mathrm{GH}}\left[\begin{array}{c}
a_{x h}^{V} \\
a_{y h}^{V} \\
a_{z h}^{V}
\end{array}\right]=\mathbf{T}_{\mathrm{GH}}\left[\begin{array}{c}
\dot{v} \\
v \dot{\theta} \\
-v \cos \theta \dot{\sigma}
\end{array}\right],
$$

where $\mathbf{T}_{\mathrm{GH}}$ is the transition matrix from the ballistic coordinate system to the ground inertial coordinate system and $a_{x h}^{V}, a_{y h}^{V}$, and $a_{z h}^{V}$ are the acceleration components of the hypersonic vehicle along the three axes in the ballistic coordinate system. The first term on the right side of equation (9) can be calculated by

$$
\frac{d}{\mathrm{~d} t}\left(\mathbf{T}_{\mathrm{GB}}\right)\left[\begin{array}{c}
u \\
v \\
w
\end{array}\right]=\left[\begin{array}{ccc}
T_{11} & T_{12} & T_{13} \\
T_{21} & T_{22} & T_{23} \\
T_{31} & T_{32} & T_{33}
\end{array}\right]\left[\begin{array}{c}
\dot{\varphi} \\
\dot{\psi} \\
\dot{\gamma}
\end{array}\right],
$$

where

$$
\begin{aligned}
T_{11}= & -\sin \varphi \cos \psi u-\cos \varphi \cos \psi \cos \gamma v+\cos \varphi \cos \psi \sin \gamma w, \\
T_{12}= & -\cos \varphi \sin \psi u+\cos \psi \sin \gamma v+\sin \varphi \sin \psi \cos \gamma v \\
& +\cos \psi \cos \gamma w-\sin \varphi \sin \psi \sin \gamma w, \\
T_{13}= & \sin \psi \cos \gamma v+\sin \varphi \cos \psi \sin \gamma v-\sin \psi \sin \gamma w+\sin \varphi \cos \psi \cos \gamma w, \\
T_{21}= & \cos \varphi u-\sin \varphi \cos \gamma v+\sin \varphi \sin \gamma w, \\
T_{22}= & 0, \\
T_{23}= & -\cos \varphi \sin \gamma v-\cos \varphi \cos \gamma w, \\
T_{31}= & \sin \varphi \sin \psi u+\cos \varphi \sin \psi \cos \gamma v-\cos \varphi \sin \psi \sin \gamma w, \\
T_{32}= & -\cos \varphi \cos \psi u-\sin \psi \sin \gamma v+\sin \varphi \cos \psi \cos \gamma v \\
& -\sin \psi \cos \gamma w-\sin \varphi \cos \psi \sin \gamma w, \\
T_{33}= & \cos \psi \cos \gamma v-\sin \varphi \sin \psi \sin \gamma v-\cos \psi \sin \gamma w-\sin \varphi \sin \psi \cos \gamma w .
\end{aligned}
$$

Combining equations (6) and (9)-(11), the following equation can be obtained:

$$
\mathbf{T}_{\mathrm{GH}}\left[\begin{array}{c}
a_{x h}^{V} \\
a_{y h}^{V} \\
a_{z h}^{V}
\end{array}\right]=\mathbf{T}_{\mathrm{GB}}\left[\begin{array}{c}
\dot{u} \\
\dot{v} \\
\dot{w}
\end{array}\right]+\mathbf{A B}\left[\begin{array}{c}
\omega_{x} \\
\omega_{y} \\
\omega_{z}
\end{array}\right],
$$

where $\mathbf{A}, \mathbf{B} \in \mathbf{R}^{3 \times 3}$ are given by

$$
\begin{aligned}
\mathbf{A} & =\left[\begin{array}{ccc}
T_{11} & T_{12} & T_{13} \\
T_{21} & T_{22} & T_{23} \\
T_{31} & T_{32} & T_{33}
\end{array}\right], \\
\mathbf{B} & =\left[\begin{array}{ccc}
0 & \sin \gamma & \cos \gamma \\
0 & \cos \gamma \sec \varphi & -\sin \gamma \sec \varphi \\
1 & -\cos \gamma \tan \varphi & \sin \gamma \tan \varphi
\end{array}\right] .
\end{aligned}
$$

According to equation (13), a three-channel analytical model of body rates can be obtained:

$$
\left[\begin{array}{c}
\omega_{x} \\
\omega_{y} \\
\omega_{z}
\end{array}\right]=(\mathbf{A B})^{-1}\left(\mathbf{T}_{\mathrm{GH}}\left[\begin{array}{c}
a_{x h}^{V} \\
a_{y h}^{V} \\
a_{z h}^{V}
\end{array}\right]-\mathbf{T}_{\mathrm{GB}}\left[\begin{array}{c}
\dot{u} \\
\dot{v} \\
\dot{w}
\end{array}\right]\right) .
$$

Based on the expected acceleration components of the hypersonic vehicle along the three axes in the ballistic coordinate system, the corresponding desired body rates of the hypersonic vehicle with respect to the roll, yaw, and pitch channels can be generated by equation (15). This analytical equation can help in replacing the process of "generating commands of angle of attack and sideslip angle/bank angle according to the desired acceleration of the hypersonic vehicle $\longrightarrow$ designing the attitude control system to track the commands of rotational Euler angles" in the traditional design scheme of the G\&C system. Besides, the integration of centroid motion model and rotational motion model can be improved, and the design procedure of the G\&C system can be simplified.

3.2. Guidance Subsystem Design. Equation (7) can be rewritten as 


$$
\left[\begin{array}{c}
\ddot{\lambda}_{D} \\
\ddot{\lambda}_{T}
\end{array}\right]=\left[\begin{array}{l}
f_{1} \\
f_{2}
\end{array}\right]+\left[\begin{array}{cc}
\frac{-1}{r} & 0 \\
0 & \frac{1}{\left(r \cos \lambda_{D}\right)}
\end{array}\right]\left[\begin{array}{c}
a_{y s}^{V} \\
a_{z s}^{V}
\end{array}\right]
$$

where

$$
\left[\begin{array}{l}
f_{1} \\
f_{2}
\end{array}\right]=\left[\begin{array}{l}
\frac{\left(-2 \dot{r} \dot{\lambda}_{D}-r \dot{\lambda}_{T}^{2} \cos \lambda_{D} \sin \lambda_{D}+a_{y s}^{T}\right)}{r} \\
\frac{\left(2 r \dot{\lambda}_{D} \dot{\lambda}_{T} \sin \lambda_{D}-2 \dot{r} \dot{\lambda}_{T} \cos \lambda_{D}-a_{z s}^{T}\right)}{\left(r \cos \lambda_{D}\right)}
\end{array}\right] .
$$

In this article, the hypersonic vehicle is steered to the ground maneuver target by zeroing $\dot{\lambda}_{D}$ and $\dot{\lambda}_{T}$. Based on equation (17), $a_{y s}^{V}$ and $a_{z s}^{V}$ are regarded as the virtual inputs to zero the angle rates of LOS. A sliding mode surface vector is conducted as $\mathbf{S}_{1}=\left[\begin{array}{ll}S_{D} & S_{T}\end{array}\right]^{T}=\left[\begin{array}{ll}\dot{\lambda}_{D} & \dot{\lambda}_{T}\end{array}\right]^{T}$, and the anticipant dynamic of $\mathbf{S}_{1}$ is chosen as

$$
\left[\begin{array}{c}
\dot{S}_{D} \\
\dot{S}_{T}
\end{array}\right]=\left[\begin{array}{c}
-\varepsilon_{D}\left|S_{D}\right|^{\rho_{D}} \operatorname{sgn}\left(S_{D}\right)-k_{D} S_{D} \\
-\varepsilon_{T}\left|S_{T}\right|^{\rho_{T}} \operatorname{sgn}\left(S_{T}\right)-k_{T} S_{T}
\end{array}\right],
$$

where $\varepsilon_{D}, \varepsilon_{T}, \rho_{D}, \rho_{T}, k_{D}$, and $k_{T}$ are parameters that need to be designed suitably to make $S_{D}$ and $S_{T}$ converge to zero gradually without chattering phenomenon.

In equation (16), $f_{1}$ and $f_{2}$ contain acceleration components of the ground maneuver target, which cannot be directly obtained by the seeker of the hypersonic vehicle. In this article, the acceleration components of the target in the LOS coordinate system are recognized as bounded uncertainties. The ESO technology is used to estimate $f_{1}$ and $f_{2}$ including uncertainties [28]:

$$
\left\{\begin{array}{l}
\dot{\mathbf{Z}}_{1}=\mathbf{Z}_{2}-\beta_{1}\left(\mathbf{Z}_{1}-\left[\begin{array}{l}
\dot{\lambda}_{D} \\
\dot{\lambda}_{T}
\end{array}\right]\right)+\left[\begin{array}{cc}
\frac{-1}{r} & 0 \\
0 & \frac{1}{\left(r \cos \lambda_{D}\right)}
\end{array}\right]\left[\begin{array}{l}
a_{y s}^{V} \\
a_{z s}^{V}
\end{array}\right], \\
\dot{\mathbf{Z}}_{2}=\beta_{2}\left(\mathbf{z}_{1}-\left[\begin{array}{l}
\dot{\lambda}_{D} \\
\dot{\lambda}_{T}
\end{array}\right]\right),
\end{array}\right.
$$

where $\beta_{1}$ and $\beta_{2}$ are parameters that need to be designed, $\mathbf{Z}_{1}$, $\mathbf{Z}_{2} \in \mathbf{R}^{2 \times 1}$ are the state vectors of the ESO, and the two elements in $\mathbf{Z}_{2}$ are the estimated values of $f_{1}$ and $f_{2}$. In practical engineering application, real-time $a_{y s}^{V}$ and $a_{z s}^{V}$ can be obtained by

$$
\left[\begin{array}{c}
a_{y s}^{V} \\
a_{z s}^{V}
\end{array}\right]=\mathbf{T}_{\mathrm{SG}, 23}\left(\mathbf{T}_{\mathrm{GB}}\left[\begin{array}{c}
a_{x b}^{V} \\
a_{y b}^{V} \\
a_{z b}^{V}
\end{array}\right]+\mathbf{A B}\left[\begin{array}{c}
\omega_{x} \\
\omega_{y} \\
\omega_{z}
\end{array}\right]\right)
$$

where $\mathbf{T}_{\mathrm{SG}, 23}$ represents the matrix reconstructed with the second and third rows of the transition matrix from the ground inertial coordinate system to the LOS coordinate system:

$$
\mathbf{T}_{\mathrm{SG}, 23}=\left[\begin{array}{ccc}
-\sin \lambda_{D} \cos \lambda_{T} & \cos \lambda_{D} & \sin \lambda_{D} \sin \lambda_{T} \\
\sin \lambda_{T} & 0 & \cos \lambda_{T}
\end{array}\right],
$$

where $\left[\begin{array}{lll}a_{x b}^{V} & a_{y b}^{V} & a_{z b}^{V}\end{array}\right]^{T}$ is the acceleration of the hypersonic vehicle in the body coordinate system and is measured by the accelerometer mounted on the hypersonic vehicle. Hence, $f_{1}$ and $f_{2}$ can be estimated and the acceleration components of the target in the LOS coordinate system can be well compensated by making full use of acceleration information of the hypersonic vehicle. It should be noted that real-time $a_{y s}^{V}$ and $a_{z s}^{V}$ in the simulation experiments are obtained based on the centroid dynamic model of the hypersonic vehicle in this article.

Combining equations (16), (18), and (19), the desired virtual inputs can be obtained by

$$
\left[\begin{array}{c}
a_{y s}^{\mathrm{V}, \mathrm{c}} \\
a_{z s}^{\mathrm{V}, \mathrm{c}}
\end{array}\right]=\left[\begin{array}{cc}
\frac{-1}{r} & 0 \\
0 & \frac{1}{\left(r \cos \lambda_{\mathrm{D}}\right)}
\end{array}\right]^{-1}\left(\left[\begin{array}{c}
\dot{S}_{\mathrm{D}} \\
\dot{S}_{\mathrm{T}}
\end{array}\right]-\mathbf{Z}_{2}\right)
$$

Let $\mathbf{C}=\mathbf{T}_{\mathrm{SG}, 23} \mathbf{T}_{\mathrm{GH}}$. Combining equations (15) and (22), the desired three-channel body rates of the hypersonic vehicle can be directly generated as

$$
\left[\begin{array}{c}
\omega_{x, c} \\
\omega_{y, c} \\
\omega_{z, c}
\end{array}\right]=(\mathbf{A B})^{-1}\left(\mathbf{T}_{\mathrm{GH}} \mathbf{C}^{-1}\left[\begin{array}{cc}
\frac{-1}{r} & 0 \\
0 & \frac{1}{\left(r \cos \lambda_{D}\right)}
\end{array}\right]^{-1}\left(\left[\begin{array}{c}
\dot{S}_{D} \\
\dot{S}_{T}
\end{array}\right]-\mathbf{Z}_{2}\right)-\mathbf{T}_{\mathrm{GB}}\left[\begin{array}{c}
\dot{u} \\
\dot{v} \\
\dot{w}
\end{array}\right]\right) .
$$


It should be noted that the change rates of the desired three-channel body rates are indispensable in the design process of the attitude control subsystem. However, differentiating the desired body rates directly is more inconvenient in engineering application and increases the amount of calculation. Hence, bașed on the dynamic surface control theory, $\left[\begin{array}{lll}\omega_{x, c} & \omega_{y, c} & \omega_{z, c}\end{array}\right]^{T}$ is passed through a low-pass filter:

$$
\tau\left[\begin{array}{c}
\dot{\omega}_{x, d} \\
\dot{\omega}_{y, d} \\
\dot{\omega}_{z, d}
\end{array}\right]+\left[\begin{array}{c}
\omega_{x, d} \\
\omega_{y, d} \\
\omega_{z, d}
\end{array}\right]=\left[\begin{array}{c}
\omega_{x, c} \\
\omega_{y, c} \\
\omega_{z, c}
\end{array}\right],
$$

where $\tau \in \mathbf{R}^{3 \times 3}$ is the designed parameter matrix of the lowpass filter. The outputs $\omega_{x, d}, \omega_{y, d}$, and $\omega_{z, d}$ are the final threechannel commands of body rates generated in the guidance subsystem. By designing the attitude controller to track the three-channel commands of body rates, $\dot{\lambda}_{D}$ and $\dot{\lambda}_{T}$ can gradually converge to zero. Hence, the hypersonic vehicle can successfully impact the ground maneuver target.

It should be noted that the inverse of matrix $\mathbf{A B}$ and the generalized inverse of matrix $\mathbf{C}$ are included in equation (23). The elements of matrix $\mathbf{A B}$ are the functions of components of velocity vector of the hypersonic vehicle in the body coordinate system and rotational Euler angles. The elements of matrix $\mathbf{C}$ are the functions of angles of LOS, flight path angle, and heading angle. As the two matrixes cannot be proved to be invertible/generalized invertible theoretically, the inverse of $\mathbf{A B}$ and the generalized inverse of $\mathbf{C}$ are assumed to exist in the whole dive phase. This assumption can only be validated by $6 \mathrm{DOF}$ simulation results.

3.3. Attitude Control Subsystem Design. To track the commands of body rates generated in the guidance subsystem, the commanded control surface fin deflections of the hypersonic vehicle should be calculated by designing the attitude control subsystem. To simplify the design process, equation (4) is rewritten as

$$
\left[\begin{array}{c}
\dot{\omega}_{x} \\
\dot{\omega}_{y} \\
\dot{\omega}_{z}
\end{array}\right]=\mathbf{D}+\mathbf{E}\left[\begin{array}{l}
\delta_{a} \\
\delta_{e} \\
\delta_{r}
\end{array}\right]
$$

where

$$
\begin{aligned}
& \mathbf{D}=\left[\begin{array}{c}
\dot{\omega}_{x}^{*} \\
\dot{\omega}_{y}^{*} \\
\dot{\omega}_{z}^{*}
\end{array}\right]=\left[\begin{array}{l}
J_{x}^{-1}\left(J_{y}-J_{z}\right) \omega_{z} \omega_{y}+J_{x}^{-1} q S l_{x} m_{x}^{*} \\
J_{y}^{-1}\left(J_{z}-J_{x}\right) \omega_{x} \omega_{z}+J_{y}^{-1} q S l_{y} m_{y}^{*} \\
J_{z}^{-1}\left(J_{x}-J_{y}\right) \omega_{y} \omega_{x}+J_{z}^{-1} q S l_{z} m_{z}^{*}
\end{array}\right], \\
& \mathbf{E}=\left[\begin{array}{ccc}
J_{x}^{-1} q S l_{x} & \\
& J_{y}^{-1} q S l_{y} & \\
& J_{z}^{-1} q S l_{z}
\end{array}\right]\left[\begin{array}{ccc}
m_{x, 1} & m_{x, 2} & m_{x, 3} \\
m_{y, 1} & m_{y, 2} & m_{y, 3} \\
m_{z, 1} & m_{z, 2} & m_{z, 3}
\end{array}\right] .
\end{aligned}
$$

In equation (25), $\delta_{a}, \delta_{e}$, and $\delta_{r}$ are the right elevon, left elevon, and rudder fin deflections of the GHV, respectively. In equation (27), $m_{i, j}, i=x, y, z, j=1,2,3$ are the coefficients related to first-degree control surface fin deflections.
In equation (26), $m_{x}^{*}, m_{y}^{*}$, and $m_{z}^{*}$ represent the remaining terms of $m_{x}, m_{y}$, and $m_{z}$ after removing the relevant parts of first-degree control surface fin deflections.

The sliding mode control method is used in the attitude control subsystem. The sliding mode surface vector is selected as

$$
\mathbf{S}_{2}=\left[\begin{array}{c}
S_{x} \\
S_{y} \\
S_{z}
\end{array}\right]=\left[\begin{array}{c}
\omega_{x}-\omega_{x, d} \\
\omega_{y}-\omega_{y, d} \\
\omega_{z}-\omega_{z, d}
\end{array}\right] .
$$

The desired dynamic of $\mathbf{S}_{2}$ is selected as

$$
\left[\begin{array}{c}
\dot{S}_{x} \\
\dot{S}_{y} \\
\dot{S}_{z}
\end{array}\right]=\left[\begin{array}{c}
-\varepsilon_{x}\left|S_{x}\right|^{\rho_{x}} \operatorname{sgn}\left(S_{x}\right)-k_{x} S_{x} \\
-\varepsilon_{y}\left|S_{y}\right|^{\rho_{y}} \operatorname{sgn}\left(S_{y}\right)-k_{y} S_{y} \\
-\varepsilon_{z}\left|S_{z}\right|^{\rho_{z}} \operatorname{sgn}\left(S_{z}\right)-k_{z} S_{z}
\end{array}\right],
$$

where $\varepsilon_{i}, \rho_{i}, k_{i}, i=x, y, z$ are parameters that need to be designed. For the attitude control subsystem, the significance of finite-time convergence is obvious. Hence, the values of $\varepsilon_{i}, \rho_{i}, k_{i}, i=x, y, z$ should be set reasonably to ensure the high convergence rate of $\mathrm{S}_{2}$ and avoid the occurrence of chattering.

Combing equations (25), (28), and (29), three-channel commands of control surface fin deflections are obtained by

$$
\left[\begin{array}{c}
\delta_{a, c} \\
\delta_{e, c} \\
\delta_{r, c}
\end{array}\right]=\mathbf{E}^{-1}\left(\left[\begin{array}{c}
\dot{S}_{x} \\
\dot{S}_{y} \\
\dot{S}_{z}
\end{array}\right]+\left[\begin{array}{c}
\dot{\omega}_{x, d} \\
\dot{\omega}_{y, d} \\
\dot{\omega}_{z, d}
\end{array}\right]-\mathbf{D}\right) .
$$

For the convenience of calculating the current commands of control surface fin deflections, $\left[\begin{array}{lll}\dot{\omega}_{x}^{*} & \dot{\omega}_{y}^{*} & \dot{\omega}_{z}^{*}\end{array}\right]^{T}$ is obtained based on the commands of control surface fin deflections generated one time step before. Define $\mathbf{x}_{1}=\left[\begin{array}{ll}\dot{\lambda}_{D} & \dot{\lambda}_{T}\end{array}\right]^{T}, \mathbf{x}_{1} \in \mathbf{R}^{2 \times 1}, \mathbf{x}_{2}=\left[\begin{array}{lll}\omega_{x} & \omega_{y} & \omega_{z}\end{array}\right]^{T}, \mathbf{x}_{2} \in \mathbf{R}^{3 \times 1}$, $\mathbf{u}=\left[\begin{array}{lll}\delta_{a} & \delta_{e} & \delta_{r}\end{array}\right]^{T}, \mathbf{u} \in \mathbf{R}^{3 \times 1}$. Based on the above design process of guidance and attitude control subsystems, the design model of the PIGC system is given by

$$
\left\{\begin{array}{l}
\dot{\mathbf{x}}_{1}=\mathbf{F}+\mathbf{G} \cdot \mathbf{x}_{2}, \\
\dot{\mathbf{x}}_{2}=\mathbf{D}+\mathbf{E} \cdot \mathbf{u},
\end{array}\right.
$$

where

$$
\begin{aligned}
& \mathbf{F}=\left[\begin{array}{c}
f_{1} \\
f_{2}
\end{array}\right]+\left[\begin{array}{cc}
\frac{-1}{r} & 0 \\
0 & \frac{1}{\left(r \cos \lambda_{D}\right)}
\end{array}\right] \mathbf{T}_{\mathrm{SG}, 23} \mathbf{T}_{\mathrm{GB}}\left[\begin{array}{c}
\dot{u} \\
\dot{v} \\
\dot{w}
\end{array}\right], \\
& \mathbf{G}=\left[\begin{array}{cc}
\frac{-1}{r} & 0 \\
0 & \frac{1}{\left(r \cos \lambda_{D}\right)}
\end{array}\right] \mathbf{T}_{\mathrm{SG}, 23} \mathbf{A B} .
\end{aligned}
$$


It is noted that the order of the design model is only 5 . Besides, the process of calculating the commands of rotational Euler angles based on the desired overload of the hypersonic vehicle in the traditional G\&C system design is elided. Hence, the design process of the proposed PIGC system exhibits the characteristic of simplification.

Taking the response delay of fin actuators into account, the generated commands of control surface fin deflections are regarded as the inputs of the second-order fin actuators. The outputs are regarded as the actual fin deflections applied to the hypersonic vehicle. The model of the fin actuators is given by

$$
\frac{\mathrm{d} \dot{\delta}_{i}}{\mathrm{~d} t}=-\omega_{n}^{2} \delta_{i}-2 \xi \omega_{n} \delta_{i}+\omega_{n}^{2} \delta_{i, c}, \quad i=a, e, r,
$$

where $\omega_{n}=20 \mathrm{~Hz}$ is the natural frequency of the secondorder model and $\xi=0.7$ is the damping ratio of the model.

\section{Simulation Tests and Discussion}

4.1. Parameter Setting. The effectiveness and robustness of the proposed PIGC method are validated by 6DOF simulation experiments based on the GHV model. The detailed model parameters and aerodynamic force and moment coefficients of GHV are provided in [29]. The maximum change rate of fin deflections is $100 \mathrm{deg} / \mathrm{s}$, and the magnitudes of fin deflections are constrained as $-20^{\circ} \leq \delta_{i} \leq+20^{\circ}, i=a, e, r$. The initial values of $6 \mathrm{DOF}$ motion states of the diving hypersonic vehicle are presented in Table 1. The values of design parameters are given in Table 2.

The ground maneuver target is considered as a point mass, and the centroid motion model is given by

$$
\left\{\begin{array}{l}
{\left[\begin{array}{lll}
\dot{x}_{T} & \dot{y}_{T} & \dot{z}_{T}
\end{array}\right]^{T}=\left[\begin{array}{lll}
v_{x T} & v_{y T} & v_{z T}
\end{array}\right]^{T},} \\
{\left[\begin{array}{lll}
\dot{v}_{x T} & \dot{v}_{y T} & \dot{v}_{z T}
\end{array}\right]^{T}=\left[\begin{array}{lll}
a_{x T} & a_{y T} & a_{z T}
\end{array}\right]^{T},}
\end{array}\right.
$$

where $\left[\begin{array}{lll}x_{T} & y_{T} & z_{T}\end{array}\right]^{T},\left[\begin{array}{lll}v_{x T} & v_{y T} & v_{z T}\end{array}\right]^{T}$, and $\left[a_{x T} a_{y T} a_{z T}\right]^{T}$ are the position, velocity, and acceleration vectors of the target in the ground inertial coordinate system. The initial components of position vector and velocity vector of the target are set as $x_{T 0}=120 \mathrm{~km}, y_{T 0}=0 \mathrm{~km}, z_{T 0}=30 \mathrm{~km}$, $v_{x T 0}=v_{y T 0}=0 \mathrm{~m} / \mathrm{s}$, and $v_{z T 0}=-25 \mathrm{~m} / \mathrm{s}$. The acceleration components of the target are set as $a_{x T}=2 \cos (0.01 t) \mathrm{m} / \mathrm{s}^{2}$, $a_{y T}=0 \mathrm{~m} / \mathrm{s}^{2}$, and $a_{z T}=1 \mathrm{~m} / \mathrm{s}^{2}$.

In this article, the longitude, latitude, and altitude of the origin of the ground inertial coordinate system are set as 0 $\mathrm{deg}, 0 \mathrm{deg}$, and $0 \mathrm{~m}$. The $o x$ axis is set to the straight east, and the $o y$ axis is set to be vertical to the ground and upward. When the altitude of the hypersonic vehicle is less than zero, the simulation test is terminated. At the terminal time, the distance between the hypersonic vehicle and target is the miss distance.

4.2. Effectiveness Verification. In order to verify the effectiveness and robustness of the proposed PIGC design method, the nominal values of aerodynamic force coefficients, aerodynamic moment coefficients, and density of atmosphere are multiplied by (1) $1+0.2 \sin (t)$, (2) $1+0.3 \sin (t)$, and (3)
TABLE 1: Initial values of $6 \mathrm{DOF}$ motion states of the hypersonic vehicle.

\begin{tabular}{lccc}
\hline States & Values & States & Values \\
\hline$v_{0}$ & $2600 \mathrm{~m} / \mathrm{s}$ & $\varphi_{0}$ & $10^{\circ}$ \\
$\theta_{0}$ & $0^{\circ}$ & $\psi_{0}$ & $-5^{\circ}$ \\
$\sigma_{0}$ & $0^{\circ}$ & $\gamma_{0}$ & $-40^{\circ}$ \\
$x_{0}$ & $0 \mathrm{~m}$ & $\omega_{x 0}$ & $0^{\circ} / \mathrm{s}$ \\
$y_{0}$ & $27000 \mathrm{~m}$ & $\omega_{y 0}$ & $0^{\circ} / \mathrm{s}$ \\
$z_{0}$ & $0 \mathrm{~m}$ & $\omega_{z 0}$ & $0^{\circ} / \mathrm{s}$ \\
\hline
\end{tabular}

TABle 2: Values of design parameters of the PIGC system.

\begin{tabular}{lccc}
\hline Parameters & Values & Parameters & Values \\
\hline$\varepsilon_{D}$ & 0.03 & $\varepsilon_{x}, \varepsilon_{y}, \varepsilon_{z}$ & 0.002 \\
$\varepsilon_{T}$ & 0.015 & $k_{x}, k_{y}, k_{z}$ & 1 \\
$k_{D}$ & 1.5 & $\rho_{x}, \rho_{y}, \rho_{z}$ & 0.6 \\
$k_{T}$ & 0.75 & $\beta_{1}$ & 200 \\
$\rho_{D}, \rho_{T}$ & 0.6 & $\beta_{2}$ & 10000 \\
$\tau$ & $\operatorname{diag}(0.04,0.04,0.04)$ & & \\
\hline
\end{tabular}

$1+0.4 \sin (t)(t$ stands for the simulation time). Simulation studies are conducted with the actual aerodynamic force and moment coefficients and atmospheric density deviating as (1)-(3). Simulation results of the three conditions are represented by red solid lines, green dash lines, and blue dot lines, respectively, as shown in Figures 2-8 .

Figure 2 presents the curves of velocity, flight path angle, and heading angle of the hypersonic vehicle in the dive phase under different deviations. As shown in Figure 2(a), the velocity magnitude of the hypersonic vehicle gradually and smoothly decreases under each deviation. It is also observed that the flight path angle and heading angle vary smoothly in the total dive phase, as illustrated in Figures 2(b) and 2(c).

Figure 3 illustrates the 3D flight trajectories of the hypersonic vehicle and curves of relative distances between the hypersonic vehicle and target under different deviations. A red pentagram indicates the location of the ground maneuver target when the simulation terminates (considering that the distances between the terminal locations of the ground maneuver target under different deviations are very short, the terminal location of the target under deviation (1) is taken as an example). It is indicated that the flight trajectories almost coincided with each other under different deviations. For deviations (1)-(3), the miss distances at the terminal time are $16.07 \mathrm{~m}, 22.55 \mathrm{~m}$, and $31.06 \mathrm{~m}$, respectively. Considering the reference length and high flight speed of the hypersonic vehicle, the miss distances under different deviations are acceptable and the proposed PIGC design method can accurately steer the hypersonic vehicle to impact the ground maneuver target with good robustness.

Figure 4 illustrates the curves of elevation and azimuth angles of LOS and change rates of angles of LOS (sliding mode surfaces $S_{D}$ and $S_{T}$ ) under different deviations. It can be observed that when the simulation time is more than 30 seconds, the change rates of elevation and azimuth angles of LOS almost converge to zero. It should be noted that when the simulation is near the end, the elevation and azimuth angles of LOS drastically change. This phenomenon is due to 


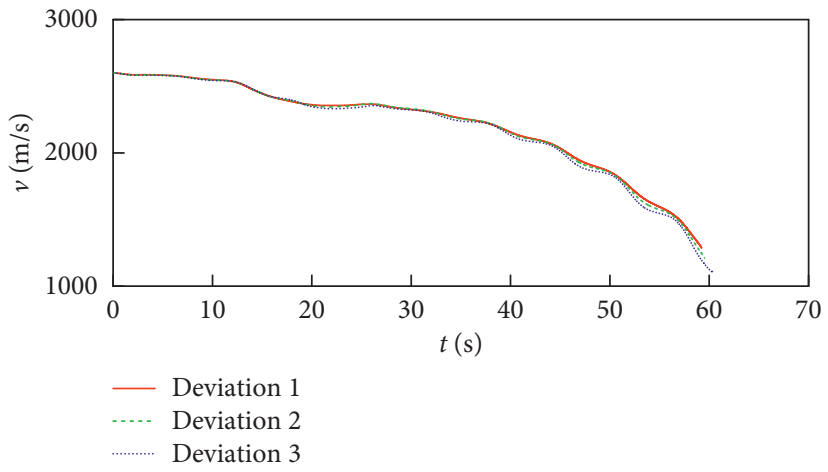

(a)

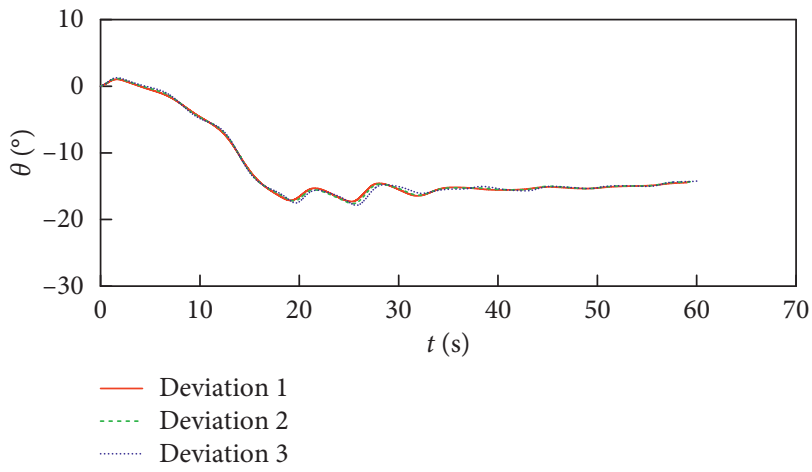

(b)

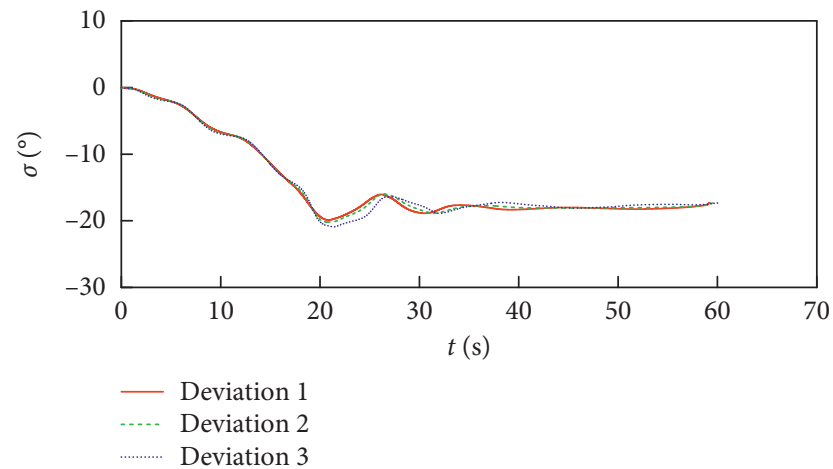

(c)

FIGURE 2: Curves of velocity, flight path angle, and heading angle.

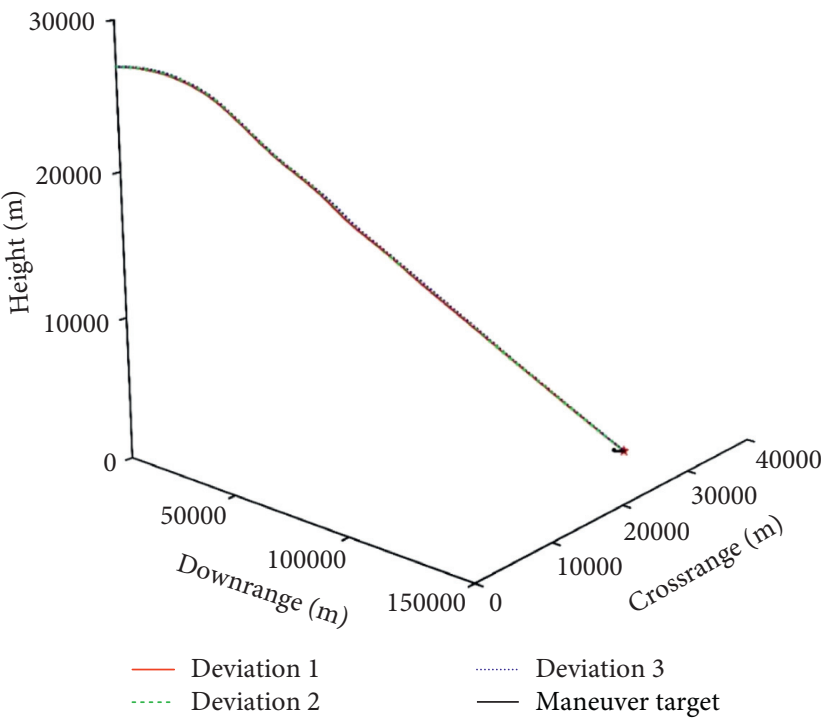

(a)

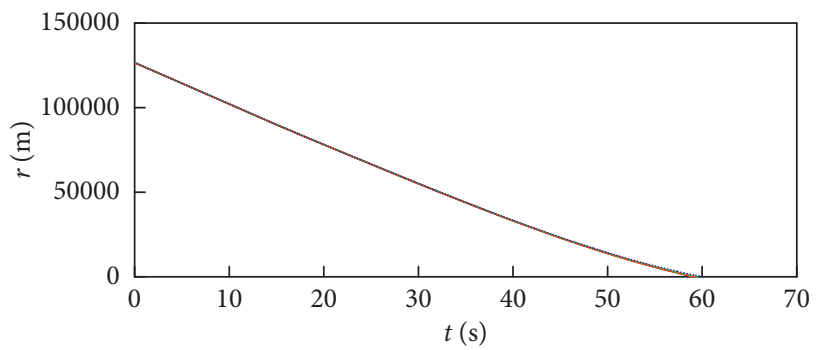

- Deviation 1

…. Deviation 2

.......... Deviation 3

Figure 3: Curves of flight trajectories of the hypersonic vehicle and relative distances between the hypersonic vehicle and target.

the fact that the two angles are calculated based on the relative spatial position of the hypersonic vehicle and target. When the distance between the hypersonic vehicle and target reduces to a small amount, the two angles drastically change.
Figures 5-7 illustrate the curves of rotational motion state variables of the hypersonic vehicle under different deviations. It is found that all attitude angles and body rates change boundedly and smoothly in the dive phase, which indicates that the attitude of the hypersonic vehicle is 


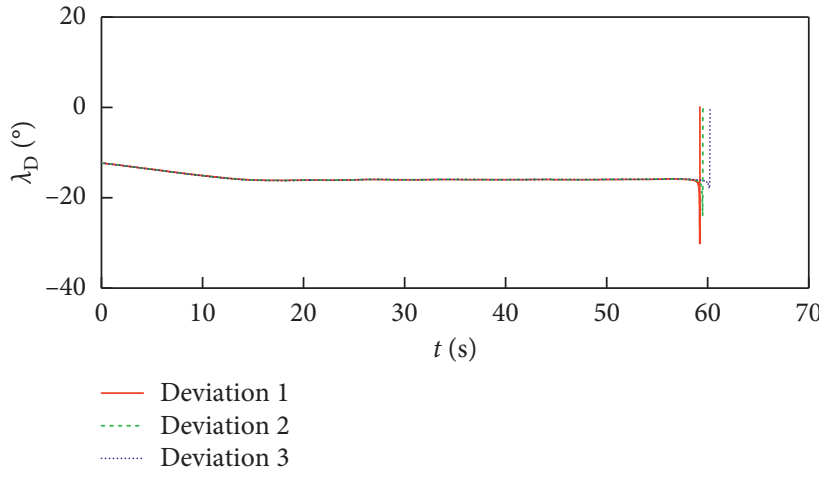

(a)

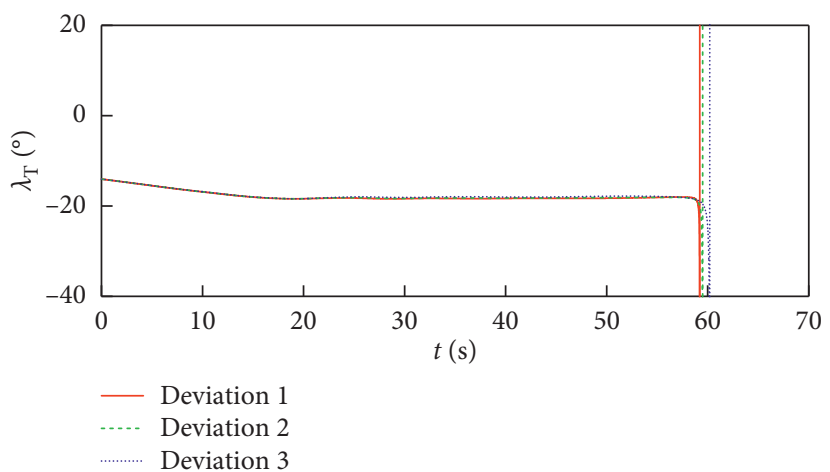

(c)

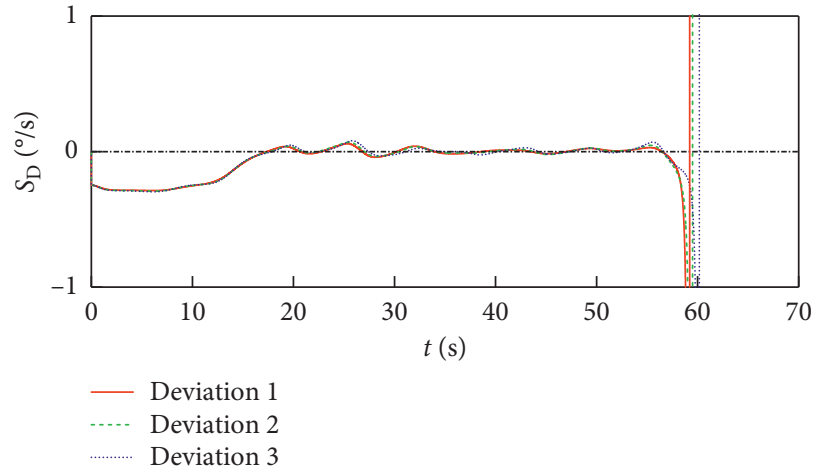

(b)

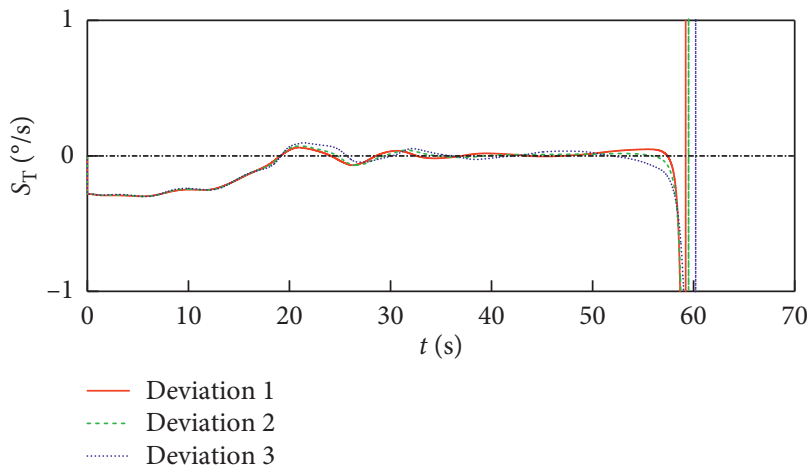

(d)

Figure 4: Curves of elevation and azimuth angles of LOS and change rates of angles of LOS.

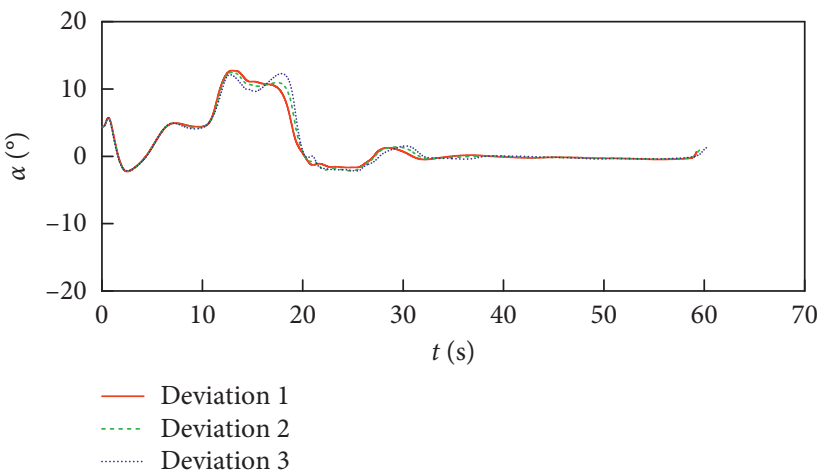

(a)

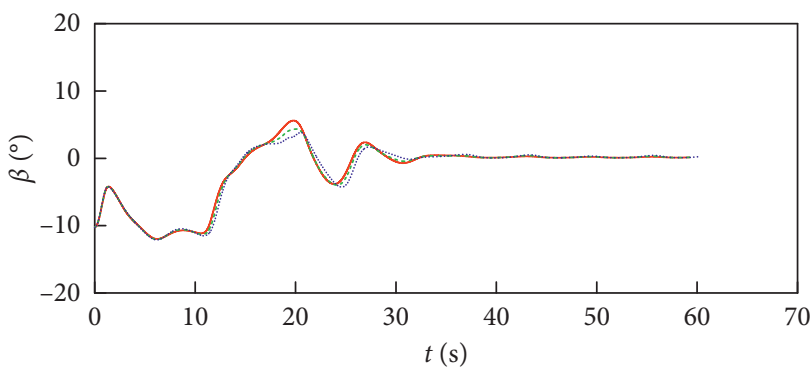

- Deviation 1

..... Deviation 2

….... Deviation 3

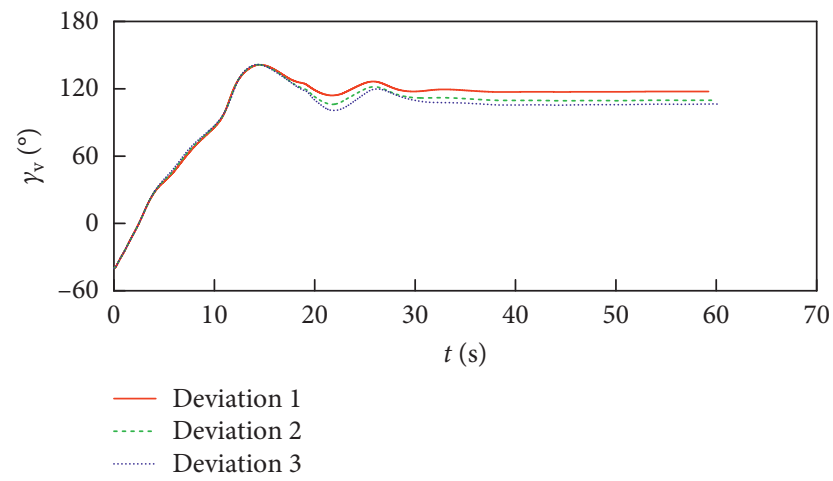

(c)

Figure 5: Curves of angle of attack, sideslip angle, and bank angle. 


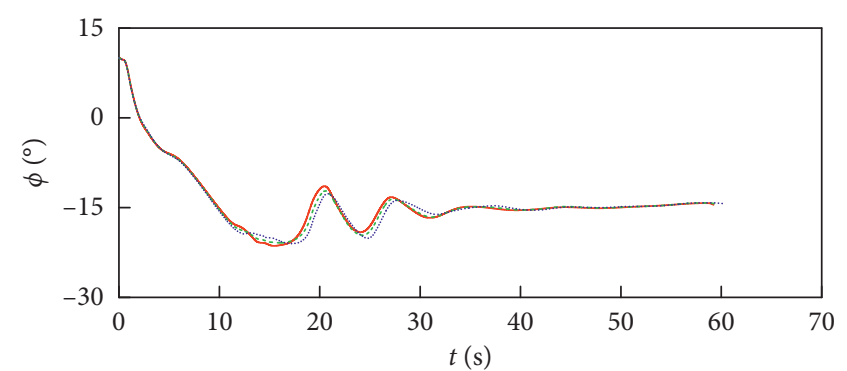

- Deviation 1

.... Deviation 2

….... Deviation 3

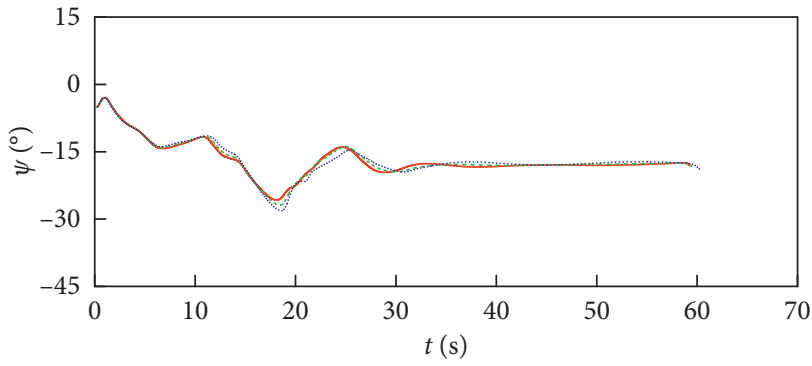

- Deviation 1

Deviation 2

Deviation 3

(a)

(b)

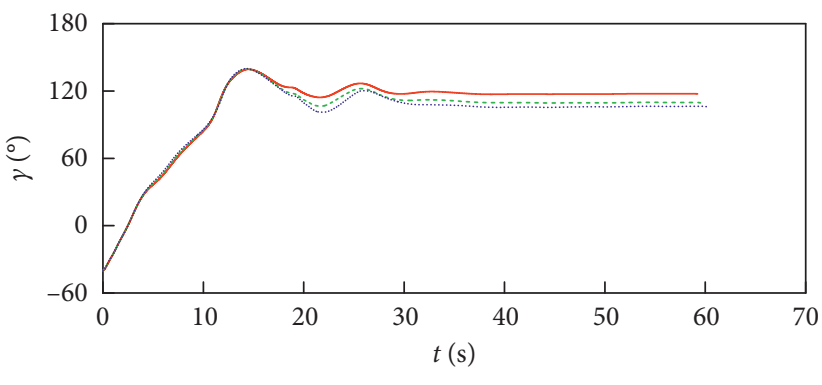

- Deviation 1

… Deviation 2

Deviation 3

(c)

FIGURE 6: Curves of pitch angle, yaw angle, and roll angle.

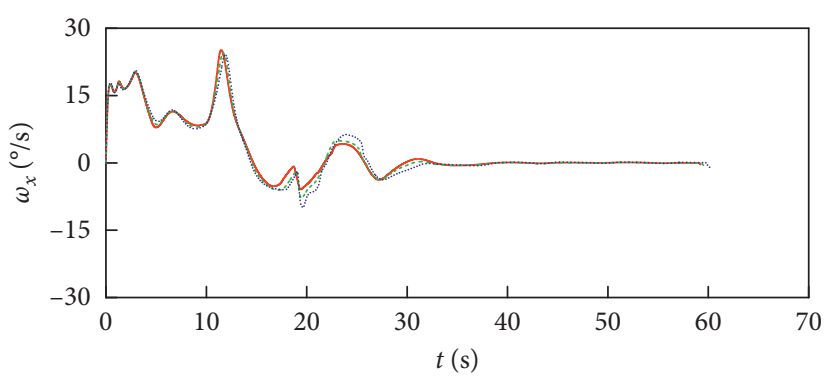

- Deviation 1

.... Deviation 2

…..... Deviation 3

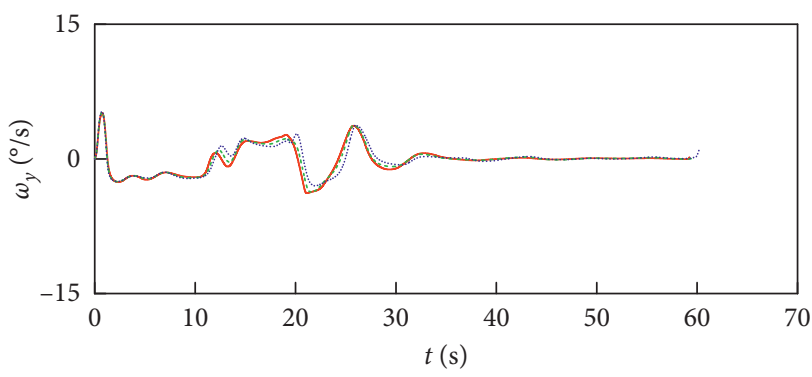

- Deviation 1

Deviation 2

…. Deviation 3

(a)

(b)

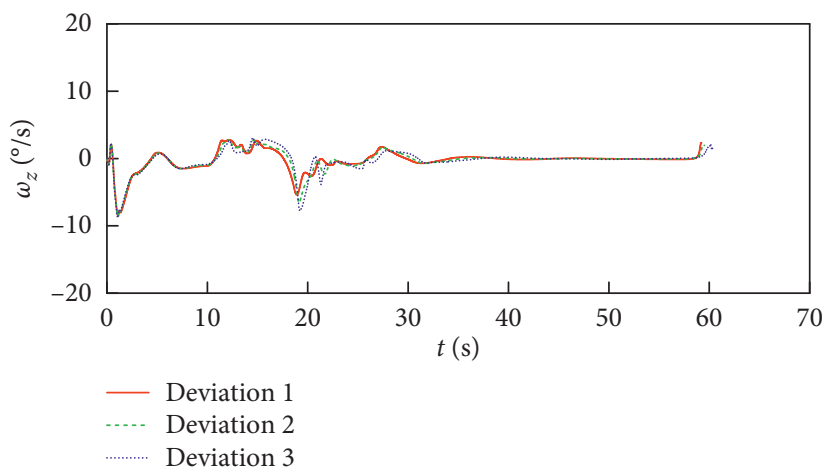

(c)

FIgURE 7: Curves of roll rate, yaw rate, and pitch rate. 


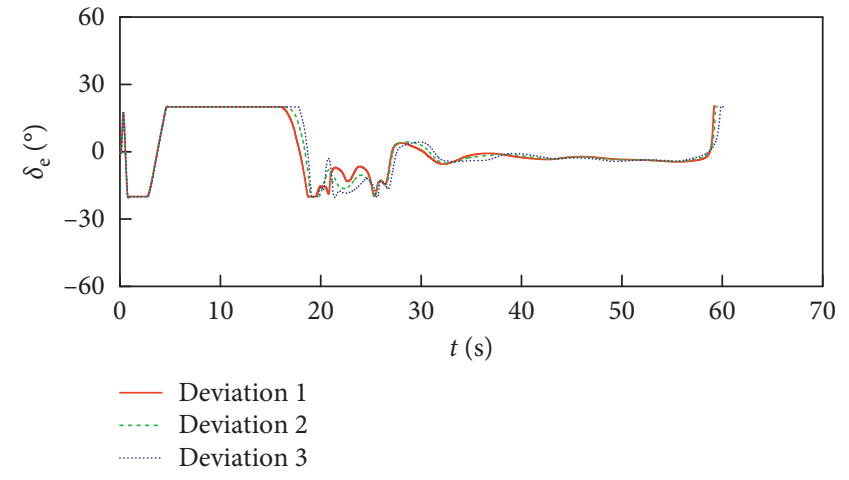

(a)

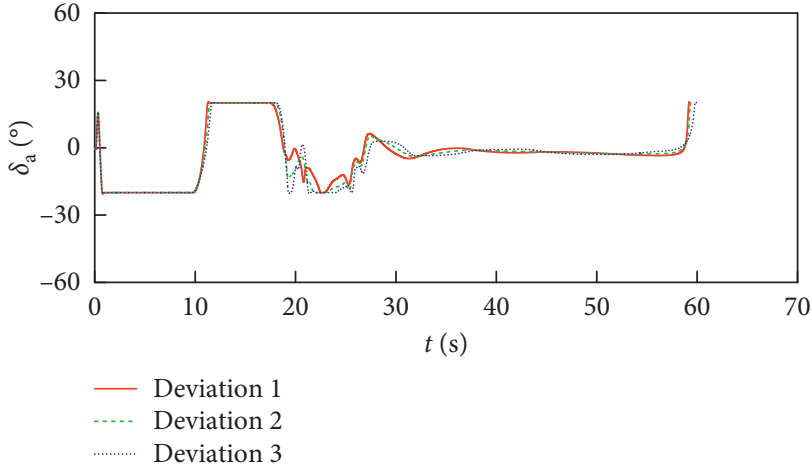

(b)

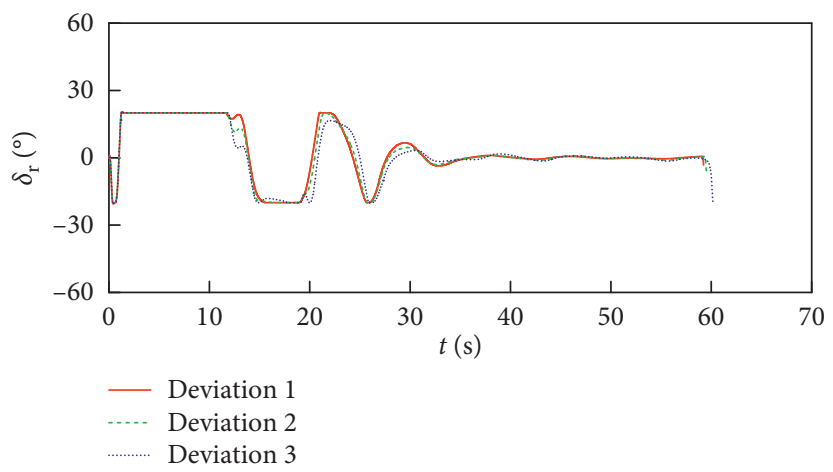

(c)

Figure 8: Curves of actual fin deflections.

controllable under each deviation. However, the change rates of rotational motion state variables are larger in the first 30 seconds of the simulation. This phenomenon is caused by the large deviation between the initial flight direction of the hypersonic vehicle and initial location of the ground maneuver target. The phenomenon is also in accordance with the fact that the elevation and azimuth angles of LOS slowly decrease in the first 30 seconds as shown in Figure 4. The three-channel body rates also exhibit similar characteristics as mentioned above.

Figure 8 illustrates the curves of actual control surface fin deflections applied to the hypersonic vehicle under different deviations, which are smooth and bounded. It is indicated that the hypersonic vehicle can complete the G\&C mission within the ability of actuators under different deviations. In addition, the assumption that the inverse of matrix $\mathbf{A B}$ and the generalized inverse of matrix $\mathbf{C}$ in equation (23) exist during the whole dive phase is validated by the simulation results.

\section{Conclusion}

In this article, a low-order PIGC method for diving hypersonic vehicles to impact ground maneuver target with good robustness is designed. A three-channel analytical model of body rates based on acceleration components of the hypersonic vehicle is deduced. By combining the analytical model of body rates and relative motion model between the hypersonic vehicle and target, three-channel commands of body rates are directly generated based on
ESO, sliding mode control approach, and dynamic surface control theory. A sliding mode controller is designed in the attitude control subsystem to track the commands of body rates. The acceleration components of the maneuver target in the relative motion model can be compensated by ESO with the use of acceleration information of the hypersonic vehicle. Hence, the utilization efficiency of the acceleration sensor mounted on the hypersonic vehicle is improved, and the impact accuracy can be guaranteed. Besides, the order of design model is reduced, and the design process of the G\&C system is simplified. However, this article is aimed at proposing a novel PIGC scheme and verifying its effectiveness and robustness. Hence, the influences of uncertainties brought by dynamic models of the hypersonic vehicle and complex constraints such as overload, dynamic pressure, and terminal impact angle are ignored, which require further study.

\section{Data Availability}

The simulation and experimental data used to support the findings of this study are available from the corresponding author upon request.

\section{Conflicts of Interest}

The authors declare that they have no conflicts of interest. 


\section{Acknowledgments}

This study was supported by the National Natural Science Foundation of China (61903379) and Research Project of State Key Laboratory of Laser Propulsion \& Application (SKLLPA-14).

\section{References}

[1] B. Xu and Z. K. Shi, "An overview on flight dynamics and control approaches for hypersonic vehicles," Science China Information Sciences, vol. 58, no. 7, pp. 1-19, 2015.

[2] J. H. Wang, L. H. Liu, P. Wang, and G. J. Tang, "Integrated guidance and control for hypersonic vehicles in dive phase," Acta Aeronautica et Astronautica Sinica, vol. 38, p. 320328, 2017.

[3] H. Yan, Research on Robust Nonlinear Integrated Guidance and Control Design, University of Science and Technology of China, Hefei, China, 2013.

[4] T. Zhao, P. Wang, L. H. Liu, and J. Wu, "Integrated guidance and control with L2 disturbance attenuation for hypersonic vehicles," Advances in Space Research, vol. 57, no. 12, pp. 2519-2528, 2016.

[5] B. Panchal, N. Mate, and S. E. Talole, "Continuous-time predictive control-based integrated guidance and control," Journal of Guidance, Control, and Dynamics, vol. 40, no. 7, pp. 1579-1595, 2017.

[6] T. Yamasaki, S. N. Balakrishnan, and H. Takano, "Separatechannel integrated guidance and autopilot for automatic path-following," Journal of Guidance, Control, and Dynamics, vol. 36, no. 1, pp. 25-34, 2013.

[7] C. Guo and X. G. Liang, "Integrated guidance and control based on block backstepping sliding mode and dynamic control allocation," Proceedings of the Institution of $\mathrm{Me}$ chanical Engineers-Part G: Journal of Aerospace Engineering, vol. 229, no. 9, pp. 1559-1574, 2015.

[8] X. L. Liang, M. Z. Hou, and G. R. Duan, “Adaptive dynamic surface control for integrated missile guidance and autopilot in the presence of input saturation," Journal of Aerospace Engineering, vol. 28, no. 5, Article ID 04014121, 2015.

[9] H. Zhou, H. Zhao, H. Q. Huang, and X. Zhao, "Integrated guidance and control design of the suicide UCAV for terminal attack," Journal of Systems Engineering and Electronics, vol. 28, no. 3, pp. 546-555, 2017.

[10] X. D. Liu, W. W. Huang, and L. F. Du, "An integrated guidance and control approach in three-dimensional space for hypersonic missile constrained by impact angles," ISA Transactions, vol. 66, pp. 164-175, 2017.

[11] W. H. Wang, S. F. Xiong, S. Wang, S. Y. Song, and C. Lai, "Three dimensional impact angle constrained integrated guidance and control for missiles with input saturation and actuator failure," Aerospace Science and Technology, vol. 53, pp. 169-187, 2016.

[12] S. Wang, W. H. Wang, and S. F. Xiong, "Impact angle constrained three-dimensional integrated guidance and control for STT missile in the presence of input saturation," ISA Transactions, vol. 64, pp. 151-160, 2016.

[13] M. Z. Hou and G. R. Duan, "Adaptive dynamic surface control for integrated missile guidance and autopilot," International Journal of Automation and Computing, vol. 8, no. 1, pp. 122-127, 2011.

[14] H. T. Song and T. Zhang, "Fast robust integrated guidance and control design of interceptors," IEEE Transactions on Control Systems Technology, vol. 24, no. 1, pp. 349-356, 2016.
[15] X. H. Wang and J. Z. Wang, "Partial integrated missile guidance and control with finite time convergence," Journal of Guidance, Control, and Dynamics, vol. 36, no. 5, pp. 13991409, 2013.

[16] X. H. Wang and J. Z. Wang, "Partial integrated guidance and control for missiles with three-dimensional impact angle constraints," Journal of Guidance, Control, and Dynamics, vol. 37, no. 2, pp. 644-657, 2014.

[17] R. Padhi, C. Chawla, and P. G. Das, "Partial integrated guidance and control of interceptors for high-speed ballistic targets," Journal of Guidance, Control, and Dynamics, vol. 37, no. 1, pp. 149-163, 2014.

[18] X. L. Shao and H. L. Wang, "Back-stepping active disturbance rejection control design for integrated missile guidance and control system via reduced-order ESO," ISA Transactions, vol. 57, pp. 10-22, 2015.

[19] C. Lai, W. H. Wang, Z. H. Liu, T. T. Liang, and S. A. Yan, "Three-dimensional impact angle constrained partial integrated guidance and control with finite-time convergence," IEEE Access, vol. 6, pp. 53833-53853, 2018.

[20] X. H. Zhou, W. H. Wang, Z. H. Liu, C. Liang, and C. Lai, "Impact angle constrained three-dimensional integrated guidance and control based on fractional integral terminal sliding mode control," IEEE Access, vol. 7, pp. 126857-126870, 2019.

[21] C. Ming, X. M. Wang, and R. S. Sun, "A novel non-singular terminal sliding mode control-based integrated missile guidance and control with impact angle constraint," Aerospace Science and Technology, vol. 94, Article ID 105368, 2019.

[22] S. Jiang, F. Q. Tian, and S. Y. Sun, "Integrated guidance and control design of rolling-guided projectile based on adaptive fuzzy control with multiple constraints," Mathematical Problems in Engineering, vol. 2019, Article ID 6309462, 17 pages, 2019.

[23] S. Jiang, F. Q. Tian, S. Y. Sun, and W. G. Liang, "Integrated guidance and control of guided projectile with multiple constraints based on fuzzy adaptive and dynamic surface," Defence Technology, vol. 16, no. 6, pp. 1130-1141, 2020.

[24] G. L. Li, T. Chao, S. Y. Wang, and M. Yang, "Integrated guidance and control for the fixed-trim vehicle against the maneuvering target," International Journal of Control, Automation and Systems, vol. 18, no. 6, pp. 1518-1529, 2020.

[25] J. Chang, Z. Y. Guo, J. Cieslak, and W. S. Chen, "Integrated guidance and control design for the hypersonic interceptor based on adaptive incremental backstepping technique," Aerospace Science and Technology, vol. 89, pp. 318-332, 2019.

[26] J. Yi, D. F. Lin, P. Pei, X. W. Shi, and W. Wang, "Robust partial integrated guidance and control approaches for maneuvering targets," International Journal of Robust and Nonlinear Control, vol. 29, pp. 6522-6541, 2019.

[27] X. L. Ai, Y. C. Shen, and L. L. Wang, "Adaptive integrated guidance and control for impact angle constrained interception with actuator saturation," Aeronautical Journal, vol. 123, no. 1267, pp. 1437-1453, 2019.

[28] Z. Zhu, D. Xu, J. M. Liu, and Y. Q. Xia, "Missile guidance law based on extended state observer," IEEE Transactions on Industrial Electronics, vol. 60, no. 12, pp. 5882-5891, 2013.

[29] S. Keshmiri, R. Colgren, and M. Mirmirani, "Six doF nonlinear equations of motion for a generic hypersonic vehicle," in Proceedings of the 2007 AIAA Atmospheric Flight Mechanics Conference and Exhibit Hilton Head, Hilton Head, South Carolina, August 2007. 